\title{
Original
}

\section{Effect of Implantable Cardioverter-defibrillator Therapy for Ventricular Fibrillation Patients with Out-of-hospital Cardiac Arrest}

\author{
Toshihiko GoKan, Mitsuharu Kawamura*, Shuhei Arai, \\ Kosuke Yoshikawa, Hiroki Tanisawa, Ko Ogawa, \\ Akinori OchI, Yoshimi Onishi, Yumi Munetsugu, \\ Hiroyuki ITO, Tatsuya ONUKI, Youichi KoBAYASHI \\ and Toshiro SHINKE
}

\begin{abstract}
Survivors of ventricular fibrillation out-of-hospital cardiac arrest (VFOHCA) due to potentially reversible causes such as acute coronary syndrome (ACS), vasospastic angina and electrolyte abnormalities are considered low risk for recurrent arrhythmia. Accordingly, implantable cardioverter-defibrillator therapy is not routinely recommended in such patients. We investigated the risk of mortality and the value of ICD therapy for VF-OHCA. Among 2,248 cardiopulmonary arrest patients presenting at our hospital, we retrospectively investigated 110 patients with VF-OHCA who were admitted for treatment. We divided the patients based on ICD $(n=71)$ or No-ICD status $(n=39)$, and on reversible cause $(n=70)$ or irreversible cause $(n=40)$. The groups were compared for baseline characteristics, mortality and ICD therapies. Patients with a reversible cause had a significantly lower rate of ICD implantation than those with an irreversible cause $(P=0.03)$. Males in the ICD group presented more frequently than those in the No-ICD group, and cardiac mortality in patients with acute coronary syndrome was significantly lower with ICD therapy than without ICD $(P=0.04)$. The rate of appropriate ICD therapies with a reversible cause was $28 \%$, and the first ICD therapy was highest within 1 year post-implantation. Patients with VF-OHCA due to a reversible cause remain at high risk of recurrent ventricular arrhythmias, suggesting that ICD implantation is a reasonable approach in such cases.
\end{abstract}

Key words : ventricular fibrillation, out-of-hospital cardiac arrest, implantable cardioverter defibrillators, sudden cardiac death

\section{Introduction}

Out-of-hospital cardiac arrest (OHCA) represents a major public health problem, affecting over 350,000 people per annum in the United States ${ }^{1)}$, with $25 \%$ of cases aged $<65$ years ${ }^{2)}$. In Japan, approximately 120,000 OHCA cases are reported annually, and while patient prognoses are improving, the recovery rate is still low even among witnessed cardiogenic cardiac arrest ${ }^{3,4)}$. An

Department of Medicine, Division of Cardiology, Showa University School of Medicine, 1-5-8 Hatanodai, Shinagawa-ku, Tokyo 142-8666, Japan.

* To whom corresponding should be addressed. 
implantable cardioverter-defibrillator (ICD) is indicated for patients who survive a sudden cardiac arrest event, with the goal of preventing mortality related to recurrent lethal ventricular arrhythmia. Indeed, current guidelines provide a Class I recommendation to implant an ICD in patients with ventricular fibrillation (VF)-OHCA or hemodynamically unstable, sustained ventricular tachycardia (VT). However, the guidelines do not recommend ICD implantation in survivors of cardiac arrest due to a potentially reversible cause, such as acute phase (24 to 48 hours) acute coronary syndrome, coronary spasm, drug-induced VF and electrolyte abnormalities $^{5-7)}$. Recently, ICD therapy was associated with lower all-cause mortality in VF-OHCA due to a reversible cause except for myocardial infarction $(\mathrm{MI})^{8)}$; however, the effectiveness of ICD use in patients with VF-OHCA due to reversible causes remains controversial. In the present study, we therefore investigated the effects of ICD therapy in patients with VF-OHCA due to a reversible cause.

\section{Methods}

\section{Patients and study protocol}

From September 2006 to December 2015, 2,248 cardiopulmonary arrest patients presented to our emergency room by ambulance. Of these, 110 surviving patients were admitted to the cardiology department due to VF-OHCA and were subsequently discharged from our hospital, and our retrospective study investigated this group of 110 patients. All patients underwent advanced cardiac life support and early defibrillation by emergency medical personnel. Patients were followed for mortality and VF/VT events for 3 years after ICD implantation. We divided the patients first into those receiving or not receiving an ICD, and then further into reversible cause and irreversible cause groups. Reversible causes were defined as ischemic heart disease, coronary spasm, drug-induced VF, electrolyte abnormalities and Takotsubo cardiomyopathy ${ }^{9,10)}$. Electrolyte abnormalities was defined as $\mathrm{K}+\leq 3.0 \mathrm{mEq} / \mathrm{l}$ or $>5.5 \mathrm{mEq} / \mathrm{l}$ or $\mathrm{Mg}^{2}+\leq 1.2 \mathrm{mEq} / \mathrm{l}$ at the first blood test. Acute MI was diagnosed based on elevation of cardiac enzymes (CK-MB or troponin $\mathrm{T}$ ) and electrocardiographic changes (new ST segment-T wave changes, new left rundle branch block or new pathologic q waves) or angiographic evidence of plaque rupture or thrombosis as per the universal definition of $\mathrm{MI}^{8)}$. Patients without evidence of acute coronary syndrome who had symptoms of myocardial ischemia prior to the arrest and angiographic evidence of significant coronary artery disease (CAD; $>75 \%$ stenosis of 1 or more major epicardial coronary arteries) were considered to have acute myocardial ischemia without infarct.

We defined coronary spasm as patients with normal coronary arteries by coronary angiography, and all of these underwent an acetylcholine provocation test more than 1 week after the initial event of cardiac arrest (performed according to the Guideline of the Japanese Circulation Society ${ }^{11)}$. Drug-induced VF was defined as the initiation of new antiarrhythmic medication one month before VF. Additionally, we defined an irreversible cause as follows: dilated cardiomyopathy (DCM), hypertrophic cardiomyopathy (HCM), hypertensive heart disease, idiopathic-VF, Brugada/J wave syndrome and QT prolongation syndrome.

Hypertensive heart disease refers to heart conditions caused by high blood pressure, including 
heart failure, thickening of the heart muscle, coronary artery disease, and other conditions are known as left ventricular hypertrophy.

The groups were compared for baseline characteristics, all-cause mortality and cardiac mortality. Furthermore, ICD therapies (appropriate and inappropriate therapy) were investigated based on the device report. Exclusion criteria were as follows: (1) patients who did not follow up with visits following hospital discharge $(n=8) ;(2)$ severe post-resuscitation encephalopathy [cerebral performance category (CPC) score $^{12}$ ) 4 or 5]; and (3) age $>90$ years. All patients provided written informed consent for the ICD therapy before device implantation, and our hospital's institutional review board approved this study.

\section{Indications for ICD implantation}

In patients with VF-OHCA due to an irreversible cause, we always recommended ICD implantation in patients without CPC 4 or 5, and only proceeded if the patients and families consented to the therapy. No ICD was implanted if patients or families rejected the therapy.

In patients with VF-OHCA due to a reversible cause, we discussed the indication for ICD implantation in patients without CPC 4 or 5 and proceeded if patients and families consented to the procedure.

\section{Measurements}

In the present study, baseline clinical characteristics and mortality were evaluated from electronic medical records and telephone interview with the patient's family. We examined the data for all-cause mortality and cardiac mortality among the patient groups and assessed the ICD therapy based on the device report, including shock and anti-tachycardia pacing (ATP) therapies. ATP was attempted with eight pulses at $88 \%$ of the measured cycle length, with a 10-ms decrement between bursts. The initial device shock was attempted at the defibrillation threshold, plus at least $10 \mathrm{~J}$. The remaining device shock should therefore represent the maximal energy shocks. ICD programming was performed as directed by the attending physician. An appropriate therapy event was defined as ATP and shock therapies being delivered for VT and VF. An inappropriate therapy event was defined as ATP and shock therapies being delivered for tachycardia [including atrial fibrillation (AF), supraventricular tachycardias (SVTs), sinus tachycardia] and device error, such as over sensing and lead dislodgement. The ejection fraction (EF) was assessed by transthoracic echocardiogram with biplane Simpson's equation using the apical 4- and 2-chamber views.

\section{Statistical analysis}

Data are presented as mean \pm SD and ANOVA was used for multiple-group comparisons. Categorical data are summarised by frequencies and percentages. The unpaired Student's t-test was used to analyse differences in baseline characteristics in the ICD and No-ICD group patients, and among patients in the reversible and irreversible groups. The paired Student's t-test was used to compare continuous data within the subgroups during follow-up. The Kaplan- 
Meier method was used to analyse the time to recurrence of the therapy event and mortality during the follow-up period. Such data were then compared using the log rank test. The Cox regression model was used to estimate the hazard ratio and its confidence intervals. We considered $P$-values $<0.05$ as statistically significant.

\section{Results}

\section{Patient characteristics}

We investigated 110 surviving patients with VF-OHCA, of whom 71 (64\%) cases were implanted with an ICD (ICD group) and 39 patients underwent no implantation (No-ICD group). Table 1 summarises all baseline characteristics of patients in the ICD group. Patients in the ICD group tended to be younger than those in the No-ICD group (53 \pm 11 years vs $66 \pm 18$ years,

Table 1. Baseline characteristics for all patients

\begin{tabular}{|c|c|c|c|}
\hline & ICD group & No-ICD group & $P$ value \\
\hline All patients $(\mathrm{n}=110)$ & $\mathrm{n}=71$ & $\mathrm{n}=39$ & \\
\hline Age $(y$, mean \pm SD $)$ & $53 \pm 11$ & $66 \pm 18$ & 0.07 \\
\hline Males n, (\%) & $59(83 \%)$ & $22(56 \%)$ & 0.04 \\
\hline Body mass index $\left(\mathrm{kg} / \mathrm{m}^{2}\right)$ & $24 \pm 7$ & $24 \pm 8$ & 0.78 \\
\hline Ejection fraction $(\%)$ & $52 \pm 10$ & $48 \pm 11$ & 0.22 \\
\hline \multicolumn{4}{|l|}{ Underlying disease $\mathrm{n},(\%)$} \\
\hline Hypertension & $38(53 \%)$ & $29(74 \%)$ & 0.15 \\
\hline Diabetes mellitus & $18(25 \%)$ & $13(33 \%)$ & 0.52 \\
\hline Hypercholesterolemia & $38(53 \%)$ & $17(43 \%)$ & 0.67 \\
\hline Chronic kidney disease & $21(29 \%)$ & $16(41 \%)$ & 0.42 \\
\hline Atrial fibrillation & $30(42 \%)$ & $16(41 \%)$ & 0.24 \\
\hline History of coronary artery disease & $15(21 \%)$ & $8(21 \%)$ & 0.62 \\
\hline CPC 1 or 2 & $58(81 \%)$ & $28(72 \%)$ & 0.52 \\
\hline \multicolumn{4}{|l|}{ Medication on discharge } \\
\hline Beta-blocker, n (\%) & $36(50 \%)$ & $19(48 \%)$ & 0.65 \\
\hline ACE inhibitor/ARB, n (\%) & $39(55 \%)$ & $28(72 \%)$ & 0.35 \\
\hline Statin, n (\%) & $30(42 \%)$ & $15(38 \%)$ & 0.61 \\
\hline Amiodarone, n (\%) & $10(14 \%)$ & $14(36 \%)$ & 0.08 \\
\hline Diuretic, n (\%) & $27(38 \%)$ & $24(61 \%)$ & 0.01 \\
\hline Ischemic heart disease & $\mathrm{n}=22$ & $\mathrm{n}=25$ & \\
\hline Any revascularization & $22(100 \%)$ & $25(100 \%)$ & 1.00 \\
\hline PCI & $21(95 \%)$ & $23(92 \%)$ & 0.82 \\
\hline $\mathrm{CABG}$ & $1(5 \%)$ & $2(8 \%)$ & 0.80 \\
\hline Complete revascularization & $17(77 \%)$ & $21(84 \%)$ & 0.35 \\
\hline Chronic total occlusion & $7(32 \%)$ & $4(16 \%)$ & 0.07 \\
\hline Multivessel disease & $12(55 \%)$ & $8(32 \%)$ & 0.08 \\
\hline
\end{tabular}

ACE: angiotensin converting enzyme, ARB: angiotensin II receptor blocker, CABG: coronary artery bypass surgery, CPC: cerebral performance category, $\mathrm{PCI}$ : percutaneous coronary intervention 
$P=0.07)$, and they presented significantly more frequently ( $83 \%$ vs $56 \%)$, while diuretic usage in the ICD group was significantly lower than that in the No-ICD group (38\% vs 61\%). There were no significant differences in neurologically favourable outcomes between the patients with or without ICD (CPC 1 or 2: $81 \%$ vs $72 \%$ ), as was the case for those undergoing percutaneous coronary intervention (PCI) and coronary artery bypass surgery (CABG); however, chronic total occlusion and multivessel disease tended to occur at a higher rate in the ICD group than in the No-ICD group. Table 2 lists underlying diseases across the patient groups and indicates that the group with reversible causes had a significantly lower rate of ICD implantation than the irreversible group $(36 / 70,51 \%$ vs $35 / 40,86 \%)$.

\section{Mortality}

Table 3 describes mortality across the patient groups. Of note, all-cause and cardiac mortality were significantly lower in the ICD vs. the No-ICD group, as was cardiac mortality in patients with ischemic heart disease/ischemia, and cardiac mortality associated with coronary spasm tended to be less frequent. Figure 1 represents our analysis of all-cause mortality, and while it was significantly lower in the ICD vs. the No-ICD group ( $\log$ rank 0.01), the risk of mortality in the No-ICD group was high before 100 days. Figure 2 also presents a Kaplan Meier curve for all-cause mortality based on the cause. Both the reversible and irreversible cause groups showed a significantly lower mortality rate in the ICD therapy patients than in those from the No-ICD group (log rank 0.04 in both cases), indicating the usefulness of ICD implantation in patients surviving VF due to reversible causes.

\section{Number of appropriate and inappropriate ICD therapies}

In the ICD group $(n=71), 38$ patients had ICD therapies, while 33 patients did not. Among

Table 2. Underlying disease of reversible and irreversible causes

\begin{tabular}{lcc}
\hline & $\begin{array}{c}\text { ICD group } \\
(\mathrm{n}=71)\end{array}$ & $\begin{array}{c}\text { No-ICD group } \\
(\mathrm{n}=39)\end{array}$ \\
\hline Reversible cause $(\mathrm{n}=70)$ & 36 & 34 \\
Ischemic heart disease & 22 & 25 \\
Coronary spasm & 13 & 4 \\
Electrolyte abnormalities / drugs & 1 & 4 \\
Takotsubo cardiomyopathy & 0 & 1 \\
& & \\
Irreversible cause (n=40) & 35 & 5 \\
Dilated cardiomyopathy & 6 & 2 \\
Hypertrophic cardiomyopathy & 7 & 0 \\
Hypertensive heart disease & 1 & 1 \\
Idiopathic VF & 9 & 0 \\
Brugada / J wave syndrome & 7 & 1 \\
QT prolongation & 5 & \\
\hline
\end{tabular}

VF: ventricular fibrillation 
Table 3. Comparison of mortality between groups

\begin{tabular}{|c|c|c|c|}
\hline & $\begin{array}{l}\text { ICD group } \\
(\mathrm{n}=71)\end{array}$ & $\begin{array}{l}\text { No-ICD group } \\
\quad(\mathrm{n}=39)\end{array}$ & $P$ value \\
\hline All-cause mortality & $5(7 \%)$ & $12(31 \%)$ & 0.001 \\
\hline Ischemic heart disease $(n=47)$ & $3 / 22(14 \%)$ & $7 / 25(28 \%)$ & 0.22 \\
\hline Coronary spasm $(\mathrm{n}=17)$ & $0 / 13(0 \%)$ & $1 / 4 \quad(25 \%)$ & 0.06 \\
\hline Electrolyte abnormalities / drugs $(\mathrm{n}=5)$ & $0 / 1 \quad(0 \%)$ & $1 / 4 \quad(25 \%)$ & 0.62 \\
\hline Dilated cardiomyopathy $(\mathrm{n}=8)$ & $1 / 6 \quad(17 \%)$ & $2 / 2 \quad(100 \%)$ & 0.03 \\
\hline Hypertensive heart disease $(\mathrm{n}=2)$ & $1 / 1 \quad(100 \%)$ & $1 / 1 \quad(100 \%)$ & NA \\
\hline Cardiac mortality & $2(2.8 \%)$ & $9(23 \%)$ & 0.001 \\
\hline Ischemic heart disease $(n=47)$ & $0 / 22(0 \%)$ & $4 / 25(16 \%)$ & 0.04 \\
\hline Coronary spasm $(n=17)$ & $0 / 13(0 \%)$ & $1 / 4 \quad(25 \%)$ & 0.06 \\
\hline Electrolyte abnormalities / drugs $(n=5)$ & $0 / 1 \quad(0 \%)$ & $1 / 4 \quad(25 \%)$ & 0.62 \\
\hline Dilated cardiomyopathy $(\mathrm{n}=8)$ & $1 / 6 \quad(17 \%)$ & $2 / 2 \quad(100 \%)$ & 0.03 \\
\hline Hypertensive heart disease $(n=2)$ & $1 / 1 \quad(100 \%)$ & $1 / 1 \quad(100 \%)$ & NA \\
\hline
\end{tabular}

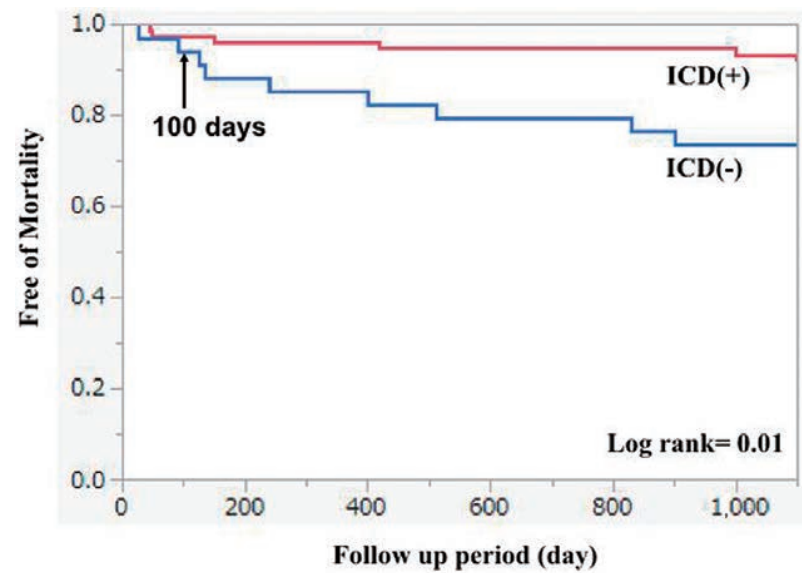

Fig. 1. Kaplan-Meier Curve for All Cause Mortality

Kaplan-Meier survival curves comparing patients with and without ICD implantation. Mortality was significantly lower in the ICD vs. the No-ICD group ( $\log$ rank 0.01), and the risk of mortality in the No-ICD group was high before 100 days post-treatment.

38 patients with ICD therapies, 25 had one or more appropriate therapies, and 15 had one or more inappropriate therapies. Two patients had both appropriate and inappropriate therapies. Among 25 patients undergoing appropriate therapies, 10 (10/36, 28\%) had a reversible cause and $15(15 / 35,45 \%)$ had an irreversible cause. Figure 3 shows a Kaplan-Meier curve representing time to first appropriate ICD therapy. The curve indicating the reversible cause data was similar to that of the irreversible cause data (log rank test 0.13). In the reversible cause group, a cumulative incidence of first ICD therapy at 1, 2 and 3 years was $20 \%, 28 \%$ and $28 \%$, respectively. The risk for first appropriate ICD therapy was highest in the 14 months post- 
A

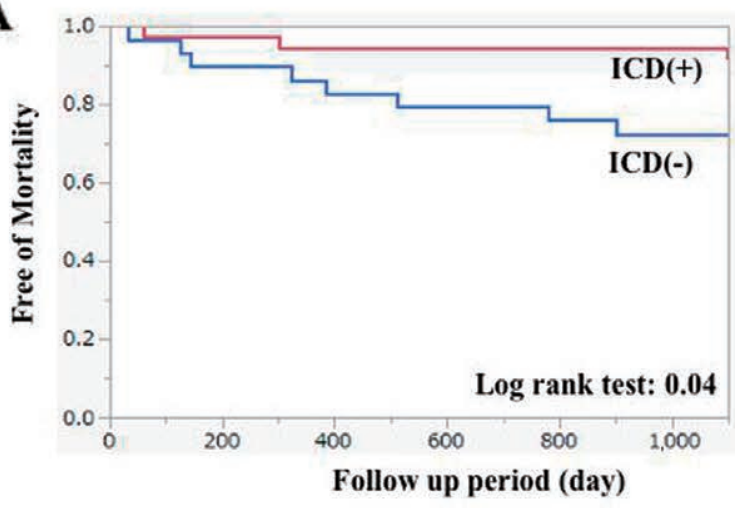

Reversible cause

Ischemic heart disease

Spasm

Hypokalemia

Drug

Takotsubo

Cardiomyopathy

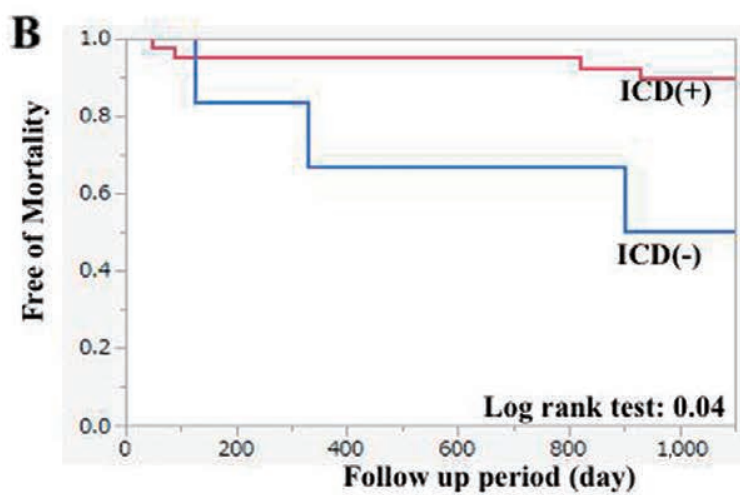

Irreversible cause

DCM

HCM

HHD

Idiopathic

Fig. 2. Kaplan-Meier analysis of all-cause mortality in the reversible (A) and irreversible groups (B). Irrespective of cause, mortality was significantly lower in the ICD group than in the No-ICD group.

DCM: dilated cardiomyopathy, HCM: hypertrophic cardiomyopathy, HHD: hypertensive heart disease.

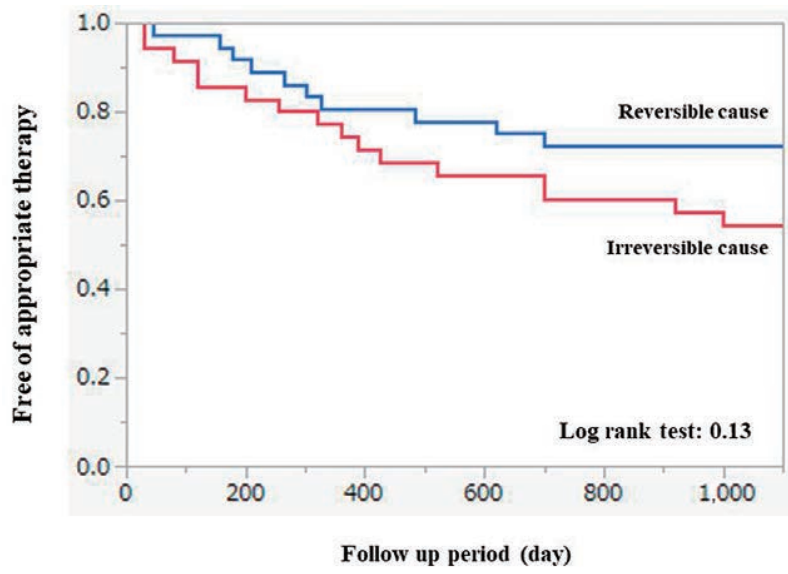

Fig. 3. Kaplan-Meier Curve for appropriate therapy of ICD Kaplan-Meier analysis of time to first appropriate ICD therapy. The curve indicated that reversible cause data was similar to that of the irreversible cause data (log rank test 0.13 ).

implantation, and this curve was similar to the mortality curve in the irreversible cause group. Table 4 compares baseline characteristics between appropriate therapy and no-appropriate therapy in the reversible cause group $(n=36)$. The ejection fraction contained in appropriate group was significantly lower relative to that for the non-appropriate group, while creatinine was significantly higher in the appropriate group than in the non-appropriate group. Patients with ischemic heart disease in the appropriate therapy group tended to rate higher rate in the non-appropriate 
Table 4. Number of appropriate and non-appropriate ICD therapies (reversible cause)

\begin{tabular}{|c|c|c|c|}
\hline & Appropriate group & Nonappropriate group & $P$ value \\
\hline All patients $(n=36)$ & $\mathrm{n}=10$ & $n=26$ & \\
\hline Age $(y)$ & 61 & 64 & 0.32 \\
\hline Male, n (\%) & $9(90 \%)$ & $22(85 \%)$ & 0.66 \\
\hline Body mass index $\left(\mathrm{kg} / \mathrm{m}^{2}\right)$ & 26 & 24 & 0.64 \\
\hline Ejection fraction $(\%)$ & $40 \pm 15$ & $51 \pm 11$ & 0.03 \\
\hline $\mathrm{BNP}(\mathrm{pg} / \mathrm{ml})$ & $851 \pm 299$ & $500 \pm 135$ & 0.22 \\
\hline Creatinine $(\mathrm{mg} / \mathrm{dl})$ & $2.86 \pm 2.83$ & $1.25 \pm 1.45$ & 0.03 \\
\hline eGFR & $45 \pm 24$ & $58 \pm 17$ & 0.08 \\
\hline Atrial fibrillation, n (\%) & $4(40 \%)$ & $10(38 \%)$ & 0.72 \\
\hline Old myocardial infarction, $\mathrm{n}(\%)$ & $3(30 \%)$ & $8(31 \%)$ & 0.61 \\
\hline \multicolumn{4}{|l|}{ Medication on discharge } \\
\hline Beta-blocker, n（\%) & $4(40 \%)$ & $13(50 \%)$ & 0.05 \\
\hline ACE inhibitor / ARB, n (\%) & $6(60 \%)$ & $20(77 \%)$ & 0.36 \\
\hline Amiodarone, n (\%) & $2(20 \%)$ & $3(11 \%)$ & 0.01 \\
\hline Ischemic heart disease $(n=22)$ & $7(70 \%)$ & $15(51 \%)$ & 0.08 \\
\hline Chronic total occlusion & $3(3 / 7,43 \%)$ & $4(4 / 15,27 \%)$ & 0.14 \\
\hline Multivessel disease & $5(5 / 7,71 \%)$ & $7(7 / 15,47 \%)$ & 0.04 \\
\hline
\end{tabular}

ACE: angiotensin converting enzyme, ARB: angiotensin II receptor blocker, BNP: brain natriuretic peptide, eGFR: estimated glomerular filtration rate

therapy group $(70 \%$ vs 51\%). Patients with multivessel disease in the appropriate therapy group presented more frequently than in the on-appropriate therapy group ( $71 \%$ vs $47 \%$ ).

\section{Discussion}

\section{Main findings}

In the present study, we retrospectively investigated the outcomes following ICD implantation for VF-OHCA due to reversible cause. The most important finding of this study is that ICD therapy significantly reduced all-cause mortality and cardiac mortality for patients with VF-OHCA due to reversible causes (i.e., ischemic heart disease, coronary spasm, drug-induced VF and electrolyte imbalance), although cardiac mortality in patients with ischemic heart disease was significantly lower with ICD as compared to those with No-ICD group when analysed in isolation. The rate of appropriate therapies with reversible cause was $28 \%$, and the first ICD therapy was highest at 1-year post-implantation.

\section{Relationship between mortality of VF-OHCA and ICD}

In trials of secondary prevention of sudden cardiac death due to irreversible cause (e.g. AVID), ICD was superior to antiarrhythmic-drug therapy in prolonging survival among patients resuscitated after symptomatic, lethal VT or VF causing hemodynamic compromise ${ }^{13)}$, with an overall mortality rate of $24.6 \%$ in the ICD group and $32 \%$ in the No-ICD group at three 
years. A Leiden out-of-hospital cardiac arrest study by Borleffs et $a l^{14)}$ also reported an overall mortality rate of $22 \%$ in the ICD group, whereas the present study showed a relatively lower mortality rate of $7 \%$ for the ICD group and $31 \%$ for the No-ICD group. Compared to previous studies, we also observed a higher average EF (50\% vs 35\%) and lower average age (50 vs 65 years). Of note, the mortality rate in our No-ICD group was similar to that in previous studies ( $31 \%$ vs $32 \%)$, as was average $\mathrm{EF}$ and age.

Reversible causes of VF-OHCA have been reported in $12-73 \%$ of cases, with an ICD implanted in $40 \%$ of survivors ${ }^{9,15)}$. In addition, current guidelines, ${ }^{5,6}$ do not recommend ICD implantation in VF-OHCA due to potentially reversible causes, including acute coronary syndrome (24 to 48 hours), coronary spasm, drug-induced VF, electrolyte abnormalities and Takotsubo cardiomyopathy. In contrast, the present study found that ICD therapy significantly reduced mortality for patients with VF-OHCA due to reversible causes, with a specific reduction rate of approximately $70 \%$. We postulated that post-resuscitation encephalopathy might be biasing the indication for ICD implantation and mortality, and herein we excluded patients with CPC 4 or 5 . Consequently, the rate of CPC 1 or 2 patients was not significantly different between the ICD and No-ICD group. Thus, post-resuscitation encephalopathy did not affect our result.

In Japan, OHCA patients with public-access defibrillation had significantly better neurological outcomes compared with those without such facilities, while no significant differences in outcomes were identified for OHCA patients in residential locations ${ }^{16)}$. ICD therapy could therefore help to reduce post-resuscitation encephalopathy with OHCA patients in residential locations, and the present data might have significant implications for the general clinical management of VF-OHCA. We always recommend ICD implantation for patients with VF-OHCA due to an irreversible cause without $\mathrm{CPC} 4$ or 5 and we often recommend ICD implantation for VF-OHCA due to a reversible cause including MI/ischemia with low EF, high creatinine and coronary spasm. These patients had more appropriate therapy and high mortality. Therefore, we feel that the current guidelines for ICD therapy in patients with VF-OHCA due to a reversible cause might need to be revised, and further studies are needed to clarify the relationship between VF-OHCA due to reversible causes and the indications for ICD implantation.

\section{ICD implantation for MI and ischemic heart disease}

The recommendations around ICD implantation for VF-OHCA due to ischemic heart disease remain controversial. Madhavan et $a l^{15)}$ reported that ICD implantation with VF-OHCA due to ischemic heart disease did not have a statistically significant association with reduced allcause mortality after adjusting for potential confounders, but showed a trend towards significance. In contrast, Ladejobi et $a l^{8)}$ reported an association between ICD therapy and lower allcause mortality except for VF-OHCA due to ischemic heart disease. In the present study, no significant difference was observed in mortality for patients with ischemic heart disease between the ICD and No-ICD groups. A previous study reported significantly lower survival rates free from major adverse cardiac events in patients with VF due to spasm compared to those without VF $(72 \% \text { vs. } 92 \% \text { at } 5 \text { years, } \mathrm{P}<0.001)^{17)}$. In our study, low EF, high concentrations 
of creatinine and ischemic heart disease patients with multivessel disease are at risk of receiving the appropriate therapies. Furthermore, cardiac mortality in the No-ICD group was significantly higher than in the group receiving ICD therapy. Therefore, we often recommend the ICD implantation for VF-OHCA due to reversible cause including ischemic heart disease with low $\mathrm{EF}$, high creatinine and multivessel disease. Yap et al ${ }^{18)}$ supported our results that coronary artery disease with low EF, high creatinine, high age and multivessel disease are at risk of mortality. Furthermore, another study indicated that patients with coronary spasm and VF were a population at high risk for recurrence of VF-OHCA, showing that ICD therapy with coronary spasm was appropriate for such populations ${ }^{19)}$. These reports also supported our study.

\section{ICD implantation for electrolyte abnormalities and Takotsubo cardiomyopathy}

In the present study, one patient with electrolyte abnormalities was implanted with an ICD and received a specific appropriate therapy during the follow-up period. Four patients with electrolyte abnormalities had no ICD implantation, and one patient (25\%) died during the followup period. In addition, a previous report ${ }^{20)}$ stated that patients with structural heart disease and an abnormal serum potassium concentration (potassium concentration $<3.0$ or $>6.0 \mathrm{mEq} / \mathrm{l}$ ) at the time of an initial episode of $\mathrm{VT} / \mathrm{VF}$ were at high risk of recurrent lethal arrhythmia $(65 \%)$. Therefore, ICD therapy might represent a reasonable choice in such clinical presentations, and our current results support this proposition.

Jesel et $a l^{21)}$ reported lethal ventricular arrhythmias occurring in approximately $10 \%$ of patients with Takotsubo cardiomyopathy, mainly in the first 24 hours of hospitalisation. Additionally, severe conduction disorders persisted during long-term follow-up. Templin et $a l^{22)}$ also reported a higher prevalence of recurrence and death in patients with Takotsubo cardiomyopathy during a 10-year follow-up. Therefore, device therapy, including ICD, might be useful for VF-OHCA due to Takotsubo cardiomyopathy.

\section{Appropriate ICD therapy for VF-OHCA due to a reversible cause}

A previous study ${ }^{15)}$ showed that the rate of ICD therapy in patients with ischemic heart disease was $35 \%$ at 3 years, and patients with $\mathrm{EF} \leq 35 \%$ at the time of discharge were at higher risk of recurrent ventricular arrhythmia. Furthermore, patients with $\mathrm{EF}>35 \%$ were declared at risk from ICD therapy in the first eight months after OHCA. In the present study, 10 patients with a reversible cause had appropriate therapies for 3 years, and 7 patients had ischemic heart disease. Furthermore, low EF, high creatinine and ischemic heart disease with multivessel disease were associated with an appropriate therapy. Our study results thus concur with previously published data, in showing that the appropriate therapy in patients with a reversible cause was approximately 30\%.

Furthermore, we observed that the risks of appropriate ICD therapy are highest in the first 14 months post-implantation, and the analysis curve was similar to the mortality curve of No-ICD in the reversible cause group. If these patients had an ICD implantation, they might have avoided lethal ventricular arrhythmias. Indeed, Epstein et $a l^{23}$ ) reported the feasibility 
and efficacy of a wearable cardiac defibrillator (WCD; an external device worn by patients capable of automatic detection and defibrillation of $\mathrm{VT} / \mathrm{VF}$ ) in high risk patients with ischemic heart disease, while another report $^{24}$ showed that implanting a subcutaneous ICD (S-ICD; a completely extra-thoracic device capable of automatic detection and defibrillation of VT/VF) might be a reasonable approach for VF-OHCA presenting without a remote ischemic heart disease and LVEF $>35 \%$. We thus propose that WCD and S-ICD could represent a good choice in VF-OHCA with $\mathrm{EF}>35 \%$, with no further escalation to ICD implantation needed in the absence of a relatively early recurrence of ventricular arrhythmia.

\section{Study limitations}

The present study has some limitations. First, it was a retrospective, nonrandomised, singlecentre study. The decision to implant an ICD was therefore likely biased by multiple factors, including age, diuretics usage and post-resuscitation encephalopathy. However, we believe that this study adequately evaluates the current clinical scenario since we identified a significant difference between mortality with VF-OHCA and ICD therapy. Furthermore, there was no significant difference in CPC between the ICD and No-ICD groups. Second, there was no clear definition of VF-OHCA due to reversible cause, thus we clearly outlined our definitions based on the AVID trial and registry. Third, this study included ischemic heart disease, coronary spasm, electrolyte abnormalities and Takotsubo cardiomyopathy. Therefore, the patients' backgrounds differed slightly, and further studies are needed to define the relationship between mortality and ICD therapy.

\section{Conclusion}

Patients with VF-OHCA due to a potentially reversible cause such as ischemic heart disease, spasm, drug/hypokalaemia or Takotsubo cardiomyopathy remain at high risk of recurrent ventricular arrhythmias. This suggests that ICD implantation might be reasonable for patients with VF-OHCA due to reversible causes including ischemic heart disease with low EF, high creatinine and multivessel disease.

\section{Conflict of interest disclosure}

All authors have no conflicts to disclose.

\section{References}

1) Lloyd-Jones D, Adams R, Carnethon M, et al. Heart disease and stroke statistics--2009 update: a report from the American Heart Association Statistics Committee and Stroke Statistics Subcommittee. Circulation. 2009;119:480-486. Erratum in: Circulation. 2009;119:e182.

2) Chugh SS, Jui J, Gunson K, et al. Current burden of sudden cardiac death: multiple source surveillance versus retrospective death certificate-based review in a large U.S. community. J Am Coll Cardiol. 2004;44:1268-1275.

3) Tahara Y. Cardiopulmonary resuscitation in a super-aging society: is there an age limit for cardiopulmonary resuscitation? Circ J. 2016;80:1102-1103. 
4) Fukuda T, Matsubara T, Doi K, et al. Predictors of favorable and poor prognosis in unwitnessed out-of-hospital cardiac arrest with a non-shockable initial rhythm. Int J Cardiol. 2014;176:910-915.

5) Epstein AE, DiMarco JP, Ellenbogen KA, et al. ACC/AHA/HRS 2008 guidelines for device-based therapy of cardiac rhythm abnormalities: a report of the American College of Cardiology/American Heart Association Task Force on Practice guidelines (writing committee to revise the ACC/AHA/NASPE 2002 guideline update for implantation of cardiac pacemakers and antiarrhythmia devices) developed in collaboration with the American Association for Thoracic Surgery and Society of Thoracic Surgeons. J Am Coll Cardiol. 2008;51:e1-e62. Erratum in: J Am Coll Cardiol. 2009;53:1473. J Am Coll Cardiol. 2009;53:147.

6) Zipes DP, Camm AJ, Borggrefe M, et al. ACC/AHA/ESC 2006 guidelines for management of patients with ventricular arrhythmias and the prevention of sudden cardiac death: a report of the American College of Cardiology/ American Heart Association Task Force and the European Society of Cardiology Committee for Practice guidelines (Writing Committee to Develop Guidelines for Management of Patients With Ventricular Arrhythmias and the Prevention of Sudden Cardiac Death). J Am Coll Cardiol. 2006;48:e247-e346.

7) JCS Joint Working Group. Guidelines for Non-Pharmacotherapy of Cardiac Arrhythmias (JCS 2011). Circ J. 2013;77:249-274.

8) Ladejobi A, Pasupula DK, Adhikari S, et al. Implantable defibrillator therapy in cardiac arrest survivors with a reversible cause. Circ Arrhythm Electrophysiol. 2018;11:e005940. (accessed 2019 Nov 22) Available from: https:// www.ahajournals.org/doi/pdf/10.1161/CIRCEP.117.005940

9) Wyse DG, Friedman PL, Brodsky MA, et al. Life-threatening ventricular arrhythmias due to transient or correctable causes: high risk for death in follow-up. J Am Coll Cardiol. 2001;38:1718-1724.

10) Thygesen K, Alpert JS, Jaffe AS, et al. Third universal definition of myocardial infarction. J Am Coll Cardiol. 2012;60:1581-1598.

11) JCS Joint Working Group. Guideline for diagnosis and treatment of patients with vasospastic angina (coronary spastic angina) (JCS 2008) : digest version. Circ J. 2010;74:1745-1762.

12) Ajam K, Gold LS, Beck SS, et al. Reliability of the Cerebral Performance Category to classify neurological status among survivors of ventricular fibrillation arrest: a cohort study. Scand J Trauma Resusc Emerg Med. 2011;19:38. (accessed 2019 Nov 22) Available from: https:/www.ncbi.nlm.nih.gov/pmc/articles/PMC3132724/pdf/1757-7241-19-38.pdf

13) Antiarrhythmics versus Implantable Defibrillators (AVID) Investigators. A comparison of antiarrhythmic-drug therapy with implantable defibrillators in patients resuscitated from near-fatal ventricular arrhythmias. $N$ Engl $J$ Med. 1997;337:1576-1583.

14) Borleffs CJ, van Erven L, Schotman M, et al. Recurrence of ventricular arrhythmias in ischaemic secondary prevention implantable cardioverter defibrillator recipients: long-term follow-up of the Leiden out-of hospital cardiac arrest study (LOHCAT). Eur Heart J. 2009;30:1621-1626.

15) Madhavan M, Friedman PA, Lennon RJ, et al. Implantable cardioverter-defibrillator therapy in patients with ventricular fibrillation out of hospital cardiac arrest secondary to acute coronary syndrome. J Am Heart Assoc. 2015;4:e001255. (accessed 2019 Nov 22) Available from: https:/www.ncbi.nlm.nih.gov/pmc/articles/PMC4345861/pdf/ jah3-4-e001255.pdf

16) Kiguchi T, Kiyohara K, Kitamura $\mathrm{T}$, et al. Public-access defibrillation and survival of out-of-hospital cardiac arrest in public vs. Residential locations in Japan. Circ J. 2019;83:1682-1688.

17) Takagi Y, Yasuda S, Tsunoda R, et al. Clinical characteristics and long-term prognosis of vasospastic angina patients who survived out-of-hospital cardiac arrest: multicenter registry study of the Japanese Coronary Spasm Association. Circ Arrhythm Electrophysiol. 2011;4:295-302.

18) Yap SC, Sakhi R, Theuns DAMJ, et al. Increased risk of ventricular arrhythmias in survivors of out-of-hospital cardiac arrest with chronic total coronary occlusion. Heart Rhythm. 2018;15:124-129.

19) Matsue Y, Suzuki M, Nishizaki M, et al. Clinical implications of an implantable cardioverter-defibrillator in patients 
with vasospastic angina and lethal ventricular arrhythmia. J Am Coll Cardiol. 2012;60:908-912.

20) Michaud GF, Sticherling C, Tada H, et al. Relationship between serum potassium concentration and risk of recurrent ventricular tachycardia or ventricular fibrillation. J Cardiovasc Electrophysiol. 2001;12:1109-1112.

21) Jesel L, Berthon C, Messas N, et al. Ventricular arrhythmias and sudden cardiac arrest in Takotsubo cardiomyopathy: incidence, predictive factors, and clinical implications. Heart Rhythm. 2018;15:1171-1178. Erratum in: Heart Rhythm. 2019;16:1598-1599.

22) Templin C, Ghadri JR, Diekmann J, et al. Clinical features and outcomes of Takotsubo (Stress) cardiomyopathy. $N$ Engl J Med. 2015;373:929-938.

23) Epstein AE, Abraham WT, Bianco NR, et al. Wearable cardioverter-defibrillator use in patients perceived to be at high risk early post-myocardial infarction. J Am Coll Cardiol. 2013;62:2000-2007.

24) Theuns DAMJ, Bhagwandien RE, Szili-Torok T, et al. Evaluation of recurrent ventricular tachyarrhythmias in patients who survived out-of-hospital cardiac arrest due to ventricular fibrillation: eligibility for subcutaneous implantable defibrillator therapy. J Interv Card Electrophysiol. 2019;55:317-323.

[Received October 30, 2019 : Accepted December 18, 2019] 